Elsevier

\title{
Plasma Postdexamethasone Cortisol Levels in Schizoaffective Disorder
}

\author{
James H. Meador-Woodruff, John F. Greden, Leon Grunhaus, and \\ Roger F. Haskett
}

Received December 21, 1987; revised version received April 6, 1988; accepted June 10, 1988.

\begin{abstract}
The degree of hypothalamic-pituitary-adrenal (HPA) axis dysregulation in depressed patients with schizoaffective disorder was compared to that seen in patients with major depressive disorder with and without delusional features. The frequency of nonsuppression to dexamethasone was similar for all three diagnostic groups. Maximum postdexamethasone plasma cortisol was greater for delusional depressives, but did not differ between patients with major depressive and schizoaffective disorders. Modest correlations were found between postdexamethasone plasma cortisol levels, severity of illness, age, and recent weight loss, for patients with both major depressive disorder and delusional depression. For schizoaffective patients, associations between postdexamethasone plasma cortisol levels and various measures of severity of illness, but not age and recent weight loss, were found. Although HPA axis dysregulation occurs more frequently in all three of the studied diagnostic groups than in normal individuals, factors contributing to this dysregulation may be qualitatively different for schizoaffective patients.
\end{abstract}

Key Words. Schizoaffective disorder, dexamethasone suppression test, depression, hypothalamic-pituitary-adrenal axis dysregulation.

Many patients with depression have dysregulation of their hypothalamic-pituitaryadrenal (HPA) axes. Early investigation suggested that this dysregulation, as reflected by nonsuppression of plasma cortisol following oral dexamethasone, might be a neuroendocrine correlate of endogenous depression (Carroll et al., 198I). Subsequently, it has become apparent that some nonendogenously depressed patients also have evidence of HPA axis dysregulation, although fewer of these patients have abnormal dexamethasone suppression test (DST) results (Mendlewicz et al., 1982; Brown et al., 1985). Thus, it seems that this dysregulation may reflect the severity of affective disturbance and is not specific for any particular subtype of depression.

A role of psychosis in HPA axis dysregulation also has been proposed. Patients with delusional depression have abnormal DST results, often demonstrating higher postdexamethasone plasma cortisol levels than depressed patients without psychosis

\footnotetext{
James H. Meador-Woodruff, M.D., is Research Track Resident; John F. Greden, M.D., is Professor and Chairman; Leon Grunhaus, M.D., is Assistant Professor; and Roger F. Haskett, M.D., is Associate Professor of Psychiatry, Dcpartment of Psychiatry, Clinical Studies Unit of the University of Michigan Depression Program, University of Michigan Medical Center, Ann Arbor, MI 48109. (Reprint requests to Dr. J.H. Meador-Woodruff, Department of Psychiatry, Mental Health Research Institute, University of Michigan Medical Center, 205 Washtenaw Place, Ann Arbor, Ml 48109, USA.)
} 
(Mendlewicz et al., 1982; Rothschild et al., 1982; Rudorfer et al., 1982; Schatzberg et al., 1983). The nature of HPA axis functioning in schizoaffective disorder is less clear; while some investigators have found high degrees of HPA disturbances in schizoaffective patients (Carman et al., 1981; Aguilar et al., 1984; Sauer et al., 1984), others have found considerably less abnormality (Greden et al., 1981; Coccaro et al., 1985; Katona and Roth, 1985). The present study was designed to explore the nature of HPA axis functioning in patients with schizoaffective disorder by examining both continuous and dichotomous postdexamethasone plasma cortisol responses in a large sample while simultaneously considering potential sources of variance known to contribute to HPA axis dysregulation.

\section{Methods}

Subjects were 84 inpatients at the Clinical Studies Unit, a 12-bed research component of the University of Michigan Depression Program. All patients were hospitalized on the unit during the period from 1977 to 1985. Diagnoses were made by consensus Research Diagnostic Criteria (RDC) (Spitzer et al., 1977) following a comprehensive diagnostic evaluation involving several unstructured clinical interviews by psychiatrists, and a structured interview by a trained research staff member using the Schedule for Affective Disorders and Schizophrenia (Spitzer and Endicott, 1975). For the present study, 49 patients had major depressive disorder (MDD), 10 MDD with psychotic features, and 25 schizoaffective disorder, depressed subtype.

After at least a 2 -week medication washout period, each patient underwent a $1 \mathrm{mg}$ DST (Carroll et al., 1981), with plasma samples obtained for cortisol determination at 8 a.m., 4 p.m., and 11 p.m. on the following day. Cortisol was determined by a modification of Murphy's (1967) competitive protein-binding technique. Intra-assay and interassay coefficients of variation in our laboratory are $5 \%$ and $7 \%$, respectively. Patients with identifiable technical exclusions known to invalidate the DST (Carroll et al., 1981) were not studied. For all patients, the 17-item Hamilton Rating Scale for Depression (HRSD) (Hamilton, 1960), and for only the schizoaffective patients, the Global Assessment Scale (GAS) and the Brief Psychiatric Rating Scale (BPRS) (Overall and Gorham, 1962), were administered at the same time that the DST was performed.

HPA axis function was compared between diagnostic groups by two methods. Patients were categorized as DST suppressors or nonsuppressors (Carroll et al., 1981), using a maximum postdexamethasone plasma cortisol level of $\geqslant 5 \mu \mathrm{g} / \mathrm{dl}$ as a referent value (Greden, 1985 ), a level that has been validated in our facility. Between-group comparisons were by $X^{2}$ analysis. In addition, absolute levels of postdexamethasone plasma cortisol levels were logarithmically transformed because the values were not normally distributed, and compared between groups by one-way analysis of variance (ANOVA). The relationships between the clinically relevant variables, severity of illness, age, and degree of recent weight change, and logarithmically transformed maximum postdexamethasone plasma cortisol levels were estimated using Pearson's product-moment correlation coefficients. All reported means are \pm standard deviations.

\section{Results}

Clinical and demographic variables for the three subgroups are shown in Table 1, and were compared between diagnostic groups by one-way ANOVA. None of the between-diagnosis differences were significant, except for age $(F=7.64, p<0.001)$; the depressed schizoaffective patients were significantly younger than patients with major depressive disorder with and without delusional features. 
Table 1. Demographic and clinical characteristics of study patients

\begin{tabular}{lccc}
\hline Variable & $\begin{array}{c}\text { MDD } \\
(\boldsymbol{n}=\mathbf{4 9 )}\end{array}$ & $\begin{array}{c}\text { MDP } \\
(\boldsymbol{n}=\mathbf{1 0})\end{array}$ & $\begin{array}{c}\text { SZD } \\
(\boldsymbol{n}=\mathbf{2 5})\end{array}$ \\
\hline Past depressive episodes & $2.6 \pm 2.9$ & $4.0 \pm 3.5$ & $2.0 \pm 2.9$ \\
Age at first depressive episode & $34.4 \pm 15.2$ & $39.5 \pm 17.4$ & $26.2 \pm 13.0$ \\
Past depressive hospitalizations & $1.8 \pm 2.1$ & $2.4 \pm 2.6$ & $1.7 \pm 2.3$ \\
Past suicide attempts & $0.8 \pm 1.8$ & $0.6 \pm 0.7$ & $1.1+1.3$ \\
Sex (male/female) & $16 / 33$ & $4 / 6$ & $9 / 16$ \\
Age & $46.2 \pm 16.3^{1}$ & $53.0 \pm 17.4^{2}$ & $33.4 \pm 10.5$ \\
Weight loss (kg) & $1.47 \pm 8.26$ & $2.86 \pm 3.53$ & $2.60 \pm 8.34$ \\
HRSD score & $21.1 \pm 5.8$ & $22.8 \pm 10.7$ & $21.2 \pm 7.9$ \\
\hline
\end{tabular}

Note. Data are presented as mean $\pm \mathrm{SD} . \mathrm{MDD}=$ major depressive disorder. MDP $=$ MDD with delusional features. $\mathrm{SZD}$ = schizoaffective disorder, depressed. HRSD = Hamilton Rating Scale for Depression.

1. MDD vs. SZD, $p<0.001$.

2. MDP vs. SZD, $p<0.005$.

Postdexamethasone plasma cortisol responses are shown in Fig. 1 and are summarized in Table 2. Delusional depressives had significantly higher plasma cortisol levels than both depressed schizoaffective patients $(F=4.83, p<0.02)$ and patients with major depressive disorder $(F=4.47, p<0.02)$. As significant betweengroup age differences were found, this analysis was also performed by analysis of covariance (ANCOVA). When age was introduced as a covariate, this difference persisted for patients with major depressive disorder $(F=3.43, p<0.05)$, but not

Fig. 1. Maximum postdexamethasone plasma cortisol levels in study population

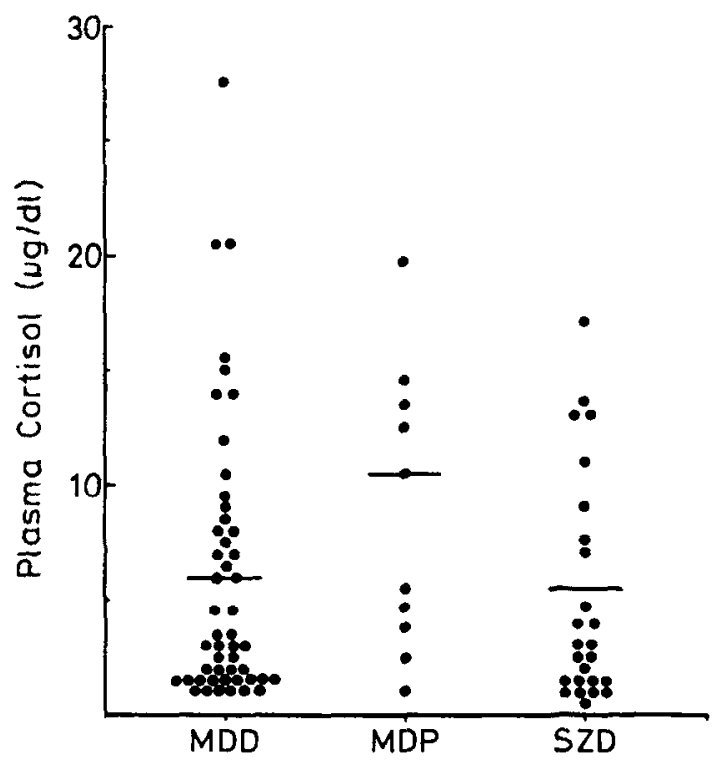

MDD $=$ major depressive disorder. MDP $=$ MDD with delusional features. SZD $=$ schizoaffective disorder, depressed subtype. 
schizoaffective disorder (depressed subtype). The percentage of DST nonsuppressors for each of the three diagnostic groups was not significantly different (Table 2).

\section{Table 2. Postdexamethasone cortisol responses in study patients}

\begin{tabular}{|c|c|c|c|}
\hline Variable & MDD & MDP & SZD \\
\hline Frequency of DST nonsuppression & $20 / 49(41 \%)$ & $6 / 10(60 \%)$ & $8 / 25(32 \%)$ \\
\hline Maximum postdexamethasone cortisol & $6.02 \pm 6.00^{2}$ & $10.58 \pm 5.50^{2}$ & $5.48 \pm 4.90^{2}$ \\
\hline Age-adjusted maximum cortisol ${ }^{1}$ & $5.73 \pm 5.60$ & $9.68 \pm 6.32^{3}$ & $6.39 \pm 6.10$ \\
\hline
\end{tabular}

Note. Results are presented as mean $\pm S D . M D D=$ major depressive disorder. MDP = MDD with delusional features. $\mathrm{SZD}=$ schizoaffective disorder, depressed. DST = dexamethasone suppression test.

1. Results of analysis of covariance, with age as covariate.

2. MDP vs. MDD and MDP vs. SZD, $p<0.02$.

3. MDP vs. MDD, $p<0.05$.

In a further attempt to characterize the response of schizoaffective patients to the DST, this group was reexamined following segregation into RDC "mainly schizophrenic" and "mainly affective" subgroups; this information, however, was only available for 15 of the 25 patients. The number of DST nonsuppressors in each subgroup was not significantly different (1/5 mainly schizophrenic and 5/10 mainly affective schizoaffective patients; $x^{2}=1.25, p=$ NS). Mean (土 SD) postdexamethasone plasma cortisol levels were also not significantly different between these two subgroups (4.1 \pm 3.9 and $5.7 \pm 4.9$ for mainly schizophrenic and affective subgroups, respectively; $F=0.41, p=\mathrm{NS}$ ). There were no differences between the two subgroups for any of the clinical and demographic variables listed in Table 1 except for age; the mainly schizophrenic schizoaffective patients tended to be somewhat younger than the mainly affective subgroup $(25.0 \pm 4.8$ vs. $36.1 \pm 8.7$ years), although this difference did not change interpretation of the cortisol data when ANCOVA was subsequently employed.

Table 3 summarizes the associations between clinically relevant variables (severity

Table 3. Coefficients of correlation between clinically relevant variables and maximum postdexamethasone plasma cortisol levels.

\begin{tabular}{lccc}
\hline Variable & $\begin{array}{c}\text { MDD } \\
(\boldsymbol{n}=\mathbf{4 9})\end{array}$ & $\begin{array}{c}\text { MDP } \\
(n=10)\end{array}$ & $\begin{array}{c}\text { SZD } \\
(n=25)\end{array}$ \\
\hline HRSD score & $0.28^{1}$ & $0.72^{1}$ & 0.34 \\
Age & $0.35^{2}$ & 0.51 & 0.03 \\
Weight loss & 0.22 & 0.53 & 0.03 \\
GAS score & & & $-0.78^{2}$ \\
BPRS score & & & $0.74^{2}$ \\
\hline
\end{tabular}

Note. $\mathrm{MDD}=$ major depressive disorder. MDP $=$ MDD with delusional features. $\mathrm{SZD}=$ schizoaffective disorder, depressed. HRSD = Hamilton Rating Scale for Depression. GAS = Global Assessment Scale. BPRS = Brief Psychiatric Rating Scale.

1. $p<0.05$.

2. $p<0.01$.

3. Due to nature of the GAS scale (increasing value implying better functioning and lower symplomatology, the reverse of the HASD and BPRS scales), an association between severity and postdexamethasone plasma cortisol would be expected to be inversely related, hence the negative $r$ value. 
of illness, age, and degree of recent weight change) and cortisol values. Moderate to high degrees of correlation were found between postdexamethasone plasma cortisol levels and measures of severity of illness (HRSD, BPRS, and GAS scores) for all three disorders. Moderate associations were found between age and degree of recent weight loss for major depressive disorder with and without delusional features, but not for schizoaffective disorder (depressed subtype).

\section{Discussion}

Previous studies on postdexamethasone cortisol responses in patients with schizoaffective disorder have focused on the dichotomous DST suppressor/nonsuppressor status, and the results have been variable. Many investigators have reported a fairly low rate of nonsuppression in schizoaffective disorder. Coccaro et al. (1985) found that only two of nine schizoaffective patients were nonsuppressors, compared with $90 \%$ of patients with psychotic depression. As these authors noted, however, the schizoaffective patients included in their study were a mainly schizophrenic rather than affective subgroup. In a previous study from our group (Greden et al., 1981), using a largely different, earlier cohort of patients, $24 \%$ of schizoaffective depressed patients were found to be DST nonsuppressors - a rate of nonsuppression between those of patients with schizophrenia and endogenous depression. Katona and Roth (1985) reported a similar nonsuppression rate of $30 \%$ in their study of 30 schizoaffective patients, although Katona and Roth's patients were not medication-free, which may confound interpretation of their results.

Other investigators have reported much higher rates of DST nonsuppression. Carman et al. (1981) indicated that $51 \%$ of the schizoaffective patients in their study were nonsuppressors, although they did not indicate their total sample size. In a study examining the diagnostic specificity of the DST, Aguilar et al. (1984) found that 9 of 13 schizoaffective patients were DST nonsuppressors, yielding a nonsuppression rate of $69 \%$. The largest DST nonsuppression rate in depressed schizoaffective patients reported to date was $89 \%$ of a study group of 18 patients (Sauer et al., 1984). Thus, the range of reported DST nonsuppression rates in depressed schizoaffective patients is from $20 \%$ to $89 \%$, indicating substantial disagreement among reports. This wide range of values may reflect the considerable heterogeneity of patients classified as schizoaffective in current nosological systems.

It is now well established that various cortisol assays may differ considerably from each other. This ensures that there will be relatively high variance in "suppression" and "nonsuppression" rates if the referent value of $5 \mu \mathrm{g} / \mathrm{dl}$ has been established in one setting (Carroll et al., 1981), but a different assay is used in other settings. Thus, a more accurate reflection of HPA regulation may be provided by examining absolute values of cortisol. Results of this type of analysis have also been variable in the study of depressed schizoaffective patients. Coccaro et al. (1985) found that patients with psychotic depression had significantly higher postdexamethasone plasma cortisol levels than patients with schizoaffective disorder, at both 4 p.m. and II p.m. Two studies have compared postdexamethasone plasma cortisol levels between depressed schizoaffective and endogenous depressive patients. In one (Greden et al., 1981), endogenous depressive patients had postdexamethasone 
plasma cortisol values twice those found in schizoaffective patients, whereas the second (Sauer et al., 1984) found that schizoaffective patients had higher cortisol levels than endogenous depressive patients. Neither of these studies, however, corrected for the possible effect of age. We found that patients with psychotic depression had significantly higher postdexamethasone plasma cortisol levels than patients with major depressive disorder and a trend to this difference persisted when results were corrected for age, a finding in agreement with many previous reports (Mendlewicz et al., 1982; Rothschild et al., 1982; Rudorfer et al., 1982; Schatzberg et al., 1983). Psychotic depressive patients also had higher cortisol levels than depressed schizoaffective patients, similar to the report of Coccaro et al. (1985); this difference did not persist, however, when age was introduced as a covariate. We found no difference between postdexamethasone plasma cortisol levels in schizoaffective disorder and major depressive disorder; unlike previous studies (Greden et al., 1981; Sauer et al., 1984), we did not restrict the group of patients with major depressive disorder to only those patients with endogenous depression.

It is apparent from these data and those from previous studies that a significant percentage of patients with schizoaffective disorder manifest some degree of HPA axis dysregulation as reflected by postdexamethasone plasma cortisol levels, but to a considerably lesser extent than that found in delusional depression. Rather, schizoaffective patients appear to have postdexamethasone plasma cortisol levels more like those of patients with major depressive disorder without psychotic features. Thus, although patients with delusional depression have the highest postdexamethasone plasma cortisol levels, the presence of psychosis in and of itself is not sufficient to account for this difference; this conclusion is further supported by previous work by Rothschild et al. (1982), who showed that psychotic schizophrenic patients did not have elevated postdexamethasone plasma cortisol levels.

Certain clinical and demographic variables have been shown to be related to the degree of HPA axis dysregulation seen in affective disorders. Age (Oxenkrug et al,, 1983; Davis et al., 1984; Lewis et al., 1984), severity of illness (Krishnan et al., 1985a; Kumar et al., 1986; Meador-Woodruff et al., 1987), and degree of recent weight change (Keitner et al., 1985; Krishnan et al., 1985b) appear to be three of the most important determinants of HPA axis dysregulation observed in depression. All three of these factors were found to be partial determinants of HPA axis dysregulation in major depressive disorder, with or without psychotic features - similar to findings in previous reports. A different pattern, however, was observed for the depressed schizoaffective patients. Severity of depression and psychosis ratings were correlated with postdexamethasone plasma cortisol levels; a modest association was observed for the depression severity ratings; and a much more significant relationship was seen for the psychosis factors. Age and weight loss, on the other hand, showed no association with plasma cortisol values. These findings suggest that although the mean postdexamethasone plasma levels might be similar in patients with major depressive and schizoaffective disorders, the factors contributing to the observed HPA axis dysregulation in these two groups may be different, perhaps reflecting differences in underlying neurotransmitter substrates. Stated differently, the underlying pathophysiology may explain both differences in clinical features and neuroendocrine findings. Additionally, severity of illness may generally be a key 
determinant of HPA axis dysregulation in various affective disorders, as it was found to be correlated with postdexamethasone cortisol levels in all three disorders studied.

The HPA axis abnormalities observed in patients with psychiatric disorders are beginning to be more fully understood. It is now accepted that they are not restricted to patients with endogenous depression. Instead, HPA axis abnormalities exist in many patients with various subtypes of affective disorders, both with and without psychotic features. Whether this HPA axis abnormality is a marker specific for affective disorders or, rather, a reflection of underlying limbic dysregulation, modified by age, weight loss, severity of symptoms, and the presence or absence of psychosis, remains to be determined.

Acknowledgment. This research was supported in part by NIMH Grant RO1-40216 (J.F.G.) and the Department of Psychiatry and the Theophile Raphael Fund at the University of Michigan Medical Center. It was presented in part at the 42nd Annual Meeting of the Society of Biological Psychiatry, Chicago, IL, May, 1987.

\section{References}

Aguilar, M.T., Lemaire, M., Castro, P., Libotte, M., Reynders, J., and Herchuelz, A. Study of the diagnostic value of the dexamethasone suppression test in endogenous depression. Journal of Affective Disorders, 6, 33 (1984).

Brown, W.A., Keitner, G., Qualls, B., and Haier, R.J. The dexamethasone suppression test and pituitary-adrenocortical function. Archives of General Psychiatry, 42, 121 (1985).

Carman, J., Wyatt, E., Crewe, E., Hall, K., Sealise, B.B.G., Watt, D. and Hoppers, L. Dexamethasone suppression test-Predictor of thymoleptic response in catatonic, paranoid, hebephrenic and schizoaffective patients. In: Perris, C., Struwe, G., and Jansson, B., eds. Biological Psychiatry: Developments in Psychiatry. Vol. 5. Elsevier, Amsterdam (1981).

Carroll, B.J., Feinberg, M., Greden, J.F., Tarika, J., Albala, A.H., Haskett, R.F., James, N.McI., Kronfol, Z., Lohr, N., Steiner, M., de Vigne, J.P., and Young, E.A. A specific laboratory test for the diagnosis of melancholia: Standardization, validation, and clinical utility. Archives of General Psychiatry, 38, 15 (1981).

Coccaro, E.F., Prudic, J., Rothpearl, A., and Nurnberg, H.G. The dexamethasone suppression test in depressive, non-depressive and schizoaffective psychosis. Journal of Affective Disorders, 9, 107 (1985).

Davis, K.L., Davis, B.M., Mathé, A.A., Mohs, R.C., Rothpearl, A.B., Levy, M.I., Gorman, L.K., and Berger, P. Age and the dexamethasone suppression test in depression. American Journal of Psychiatry, 141, 872 (1984).

Greden, J.F. Biological laboratory tests in psychiatry. In: Sadock, B.J., and Kaplan, H.I., eds. Comprehensive Textbook of Psychiatry IV. 4th ed. Williams \& Wilkins, Baltimore (1985).

Greden, J., Kronfol, Z., Gardner, R., Feinberg, M., and Carroll, B.J. Neuroendocrine evaluation of schizoaffectives with the dexamethasone suppression test. In: Perris, C., Struwe, G., and Jansson, B., eds. Biological Psychiatry: Developments in Psychiatry. Vol. 5. Elsevier, Amsterdam (1981).

Hamilton, M. A rating scale for depression. Journal of Neurology, Neurosurgery and Psychiatry, 23, 56 (1960).

Katona, C.L.E., and Roth, M. The dexamethasone suppression test in schizoaffective depression. Journal of Affective Disorders, 8, 107 (1985).

Keitner, G.I., Brown, W.A., Qualls, C.B., Haier, R.J., and Barnes, K.T. Results of the dexamethasone suppression test in psychiatric patients with and without weight loss. American Journal of Psychiatry, 142, 246 (1985). 
Krishnan, K.R.R., France, R.D., Pelton, S., McCann, U.D., Manepalli, A.N., and Davidson, J.R.T. What does the dexamethasone suppression test identify? Biological Psychiatry, 20,957 (1985a).

Krishnan, K.R.R., France, R.D., Snipes, M.T., and Pelton, S. Weight change and the dexamethasone suppression test. Biological Psychiatry, 20, 1018 (1985b).

Kumar, A., Alcser, K., Grunhaus, L., and Greden, J.F. Relationships of the dexamethasone suppression test to clinical severity and degree of melancholia. Biological Psychiatry, 21, 436 (1986).

Lewis, D.A., Pfohl, B., Schlechte, J., and Coryell, W. Influence of age on the cortisol response to dexamethasone. Psychiatry Research, 13, 213 (1984).

Meador-Woodruff, J.H., Haskett, R.F., Grunhaus, L., Akil, H., Watson, S.J., and Greden, J.F. Postdexamethasone plasma cortisol and $\beta$-endorphin levels in depression: Relationship to severity of illness. Biological Psychiatry, 22, 1137 (1987).

Mendlewicz, J., Charles, G., and Franckson, J.M. The dexamethasone suppression test in affective disorder: Relationship to clinical and genetic subgroups. British Journal of Psychiatry, 141, 464 (1982).

Murphy, B.E. Some studies of the protein-binding of steroids and their application to the routine micro- and ultra-micro measurement of various steroids in body fluids by competitive protein-binding radioassay. Journal of Clinical Endocrinology and Metabolism, 27, 973 (1967).

Overall, J.E., and Gorham, D.R. The Brief Psychiatric Rating Scale. Psychological Reports, 10, 799 (1962).

Oxenkrug, G.F., Pomara, N., McIntyre, I.M., Branconnier, R.J., Stanley, M., and Gershon, S. Aging and cortisol resistance to suppression by dexamethasone: A positive correlation. Psychiatry Research, 10, 125 (1983).

Rothschild, A.J., Schatzberg, A.F., Rosenbaum, A.H., Stahl, J.B., and Cole, J.O. The dexamethasone suppression test as a discriminator among subtypes of psychotic patients. British Journal of Psychiatry, 141, 471 (1982).

Rudorfer, M.V., Hwa, H.-G., and Clayton, P.J. Dexamethasone suppression test in primary depression: Significance of family history and psychosis. Biological Psychiatry, 17, 41 (1982).

Sauer, H., Koehler, K.G., Sass, H., Hornstein, C., and Minne, H.W. The dexamethasone suppression test and thyroid stimulating hormone response to TRH in RDC schizoaffective patients. European Archives of Psychiatry and Neurological Sciences, 234, 264 (1984).

Schatzberg, A.F., Rothschild, A.J., Stahl, J.B., Bond, T.C., Rosenbaum, A.H., Lofgren, S.B., MacLaughlin, R.A., Sullivan, M.A., and Cole, J.O. The dexamethasone suppression test: Identification of subtypes of depression. American Journal of Psychiatry, 140, 88 (1983).

Spitzer, R.L., and Endicott, J. Schedule for Affective Disorders and Schizophrenia. Biometrics Research Division, New York State Psychiatric Institute, New York (1975).

Spitzer, R.L., Endicott, J., and Robins, E. Research Diagnostic Criteria (RDC). Biometrics Research Division, New York State Psychiatric lnstitute, New York (1977). 